\title{
Optimización del juego tres en raya con niveles de dificultad utilizando heurísticas de inteligencia artificial
}

\section{Optimizing the stages of difficulty of the Tic-Tac-Toe game using artificial intelligence heuristics}

César Javier Villacís ${ }^{1}$, Walter Marcelo Fuertes ${ }^{1}$, Christian Andrés Bustamante ${ }^{2}$, Margarita Elizabeth Zambrano ${ }^{1}$, Edgar Porfirio Torres ${ }^{1}$, Hernán Mauricio Aules ${ }^{1}$, Ana Gladys Tacuri ${ }^{1}$, Mario Oswaldo Basurto ${ }^{3}$

\author{
${ }^{1}$ Universidad de las Fuerzas Armadas (ESPE), Sangolquí, Ecuador \\ ${ }^{2}$ Secretaría de Inteligencia del Gobierno Ecuatoriano, Quito, Ecuador \\ ${ }^{3}$ Universidad Tecnológica Israel, Quito, Ecuador
}

Correspondência para/Correspondence to: \{cjvillacis, wmfuertes, mezambrano, eptorres3, hmaules, agtacuri\}@espe.edu.ec, obasurto@ uisrael.edu.ec, christian.bustamante@sin.gob.ec

Recebido/Submitted: 30 Out. 2014

Aceito/Approved: 15 Nov. 2014

Copyright (C) 2014 Villacís et al. Todo o conteúdo da Revista está sob uma licença Creative Commons Atribuição-NãoComercial-Compartilhalgual 3.0 Não Adaptada. Ao serem publicados por esta Revista, os artigos são de livre uso em ambientes educacionais, de pesquisa e não comerciais, com atribuição de autoria obrigatória. Mais informações em http://www.atoz.ufpr.br/index.php/atoz/about/submissions\#copyrightNotice.

\section{Resumen}

Introducción: Los videojuegos educativos, además de proporcionar distracción y diversión, estimulan el desarrollo del pensamiento de los niños en las áreas lógica y espacial. Esta investigación presenta la optimización de un videojuego educativo relacionado con los juegos de lógica, conocido como el Tres en Raya o Tic-Tac-Toe (en inglés), enfocado a niños entre 7 y 11 años.

Método: Para llevarlo a cabo, se ha empleado el Método de Diseño Hipermedia Orientada a Objetos para el diseño conceptual y navegacional de la aplicación, lo cual permite crear interfaces de usuario interactivas y amigables con el usuario. Además, se utilizó técnicas de Inteligencia Artificial para que el jugador, simulado y controlado por la computadora, pueda emular la toma de decisiones por sí mismo, y esté en capacidad de enfrentar al usuario. Las técnicas de Inteligencia Artificial utilizadas son del tipo heurísticas, implementadas mediante un método numérico inédito, basado en series numéricas finitas, lo cual difiere de otros del mismo tipo, donde lo común es utilizar el Algoritmo del MINMAX. Para la ejecución del juego se ha recurrido a la incorporación de un agente virtual que brindará soporte al usuario en los diferentes niveles de dificultad del juego.

Resultados: Los resultados muestran que existen diferencias cognitivas por edad, género y nivel de dificultad en el juego de una muestra representativa de niños de una unidad educativa.

Conclusión: Eses tipo de programa estimula el desarrollo cognitivo de los niños que se encuentran en su etapa primaria de formación.

Palabras-clave: Videojuegos educativos. Diseño Hipermedia Orientado a Objetos. Inteligencia Artificial. Tres en Raya.

\begin{abstract}
Introduction: Educational games, in addition to providing distraction and fun, stimulate the development of children's thinking in logic and spatial areas. This research presents the optimization of an educational video game related games logic, known as Noughts and Crosses or Tic-Tac-Toe, focused on children between 7 and 11 years.

Method: To carry this out, we used the Object Oriented Hypermedia Design method for conceptual design and navigational application, which allows creating interactive and user friendly interfaces. In addition, Artificial Intelligence techniques used for the player simulated as well as controlled by computer, can emulate decisions for itself and be able to face the user. These Artificial Intelligence techniques are of heuristic type, implemented through an unprecedented numerical method based on finite numerical series, which differs from others of the same type, where it is common to use the algorithm of MIN-MAX. A virtual agent, that provides support to the users at different levels of difficulty of the game, was incorporated.

Results: The results obtained show that there are cognitive differences by age, gender and level of difficulty in the game from a representative sample of children of an educational unit.

Conclusion: This kind of program stimulates cognitive development in children who are in their primary stage of educational training.
\end{abstract}

Keywords: Educational Games. Object-Oriented Hypermedia Design Method. Artificial Intelligence. Tic-Tac-Toe.

\section{INTRODUCCIÓN}

El juego Tres en Raya estimula la cognición de los niños. Probablemente es el juego más difundido, y sencillo en su concepción, en el cual un jugador gana si consigue tener una línea recta de tres de sus símbolos del mismo tipo. La línea puede ser horizontal, vertical o diagonal. Es uno de los juegos clásicos que fueron creados en el Medio Oriente, para el desarrollo de los niños, motivándoles su destreza y habilidad mental que coadyuva a un mejor desarrollo. Normalmente son los niños pequeños los que juegan al Tres en Raya. La misma simplicidad del juego lo hace ideal como herramienta pedagógica para enseñar los conceptos de teoría de juegos.

Existen varios métodos para resolver el problema del Tres en Raya tales como el algoritmo del Minimax (Russell \& Norvig, 2002), el algoritmo de búsqueda Alpha-Beta (Watson, 2008), algoritmos genéticos (Bhatt, Varshney, \& Deb, 2008), redes neuronales (Grim, Somol, \& Pudil, 2005), Inteligencia Artificial basada en estrategias (Chakraborty, 2009), entre los más conocidos. La mayoría de estos métodos tienen un alto grado de dificultad en la resolución de este juego. Ante este escenario, esta investigación intenta encontrar una solución menos compleja basada en un sistema de reglas combinadas con técnicas heurísticas, aleatoriedad y una máquina de estados finitos representada por una lista enlazada. 
Diferentes estudios han demostrado que el juego de Tres en Raya requiere pensamiento creativo, actitud para solucionar problemas, capacidad para adquirir nuevas destrezas y habilidad para usar herramientas de software. Desde este enfoque surge el siguiente cuestionamiento ¿El juego de Tres en Raya, con diferentes niveles de dificultad, incrementa la estimulación del pensamiento cognitivo en el área lógica y espacial de los niños entre 7 y 11 años? Las preguntas orientadoras de la investigación en cambio son las siguientes: ¿Cuáles son las principales teorías educativas que fundamentan el uso de videojuegos educativos para el desarrollo cognitivo de los niños? ¿Cuál es la metodología más adecuada para diseñar, implementar y poner en funcionamiento videojuegos educativos? ¿Qué modelo matemático se puede aplicar para resolver el juego del Tres en Raya? ¿Cómo se puede evaluar y validar el videojuego educativo propuesto?

Como alternativa de solución, esta investigación presenta la implementación optimizada del video juego de Tres en Raya, enfocado a niños entre 7 y 11 años. Para llevarlo a cabo se ha empleado el Método de Diseño Hipermedia Orientada a Objetos (Object-Oriented Hypermedia Design Method - OOHDM) según Schwabe, Rossi, \& Barbosa (1996) para el diseño conceptual y navegacional de la aplicación, lo cual ha permitido crear interfaces de usuario interactivas y amigables con el usuario. Además se utilizó técnicas de Inteligencia Artificial (IA) para que el jugador simulado y controlado por la computadora pueda tomar decisiones por sí mismo y pueda enfrentarse al usuario. Las técnicas de IA utilizadas son del tipo heurísticas implementadas mediante un método numérico inédito basado en series numéricas finitas, lo cual difiere de otros del mismo tipo, donde lo común es utilizar el Algoritmo del MIN-MAX. Para su ejecución se ha recurrido a la incorporación de un agente virtual que brindará soporte al usuario en los diferentes niveles de dificultad del juego. Los resultados muestran que este tipo de programas estimulan el desarrollo cognitivo de los niños que se encuentran en su etapa primaria de formación.

Entre las principales contribuciones de este estudio se puede mencionar: (1) obtener un sistema de reglas combinadas de razonamiento con técnicas heurísticas, aleatoriedad y una máquina de estados finitos representada por una lista enlazada; (2) diseño y construcción de una aplicación con una interface gráfica de usuario (GUI) con Programación Orientada a Objetos (POO); (3) Implementación de una librería de clases que permiten representar el ambiente y las reglas del juego del Tres en Raya.

El resto del artículo ha sido organizado como sigue: La sección Marco Referencial describe las teorías que sustentan esta investigación. La sección Diseño e Implementación detalla el modelo de casos, la especificación de requerimientos, la implementación de algoritmos del juego y la implementación de su interfaz gráfica. En la siguiente sección se muestran y se discuten los resultados obtenidos. En la sección Trabajos Relacionados se analiza el estado del arte. Finalmente, se presentan las conclusiones y posibles trabajos futuros sobre la base de los resultados obtenidos.

\section{MARCO REFERENCIAL}

El juego del Tres en Raya es un juego de estrategia en el que participan dos jugadores que se enfrentan entre sí, en un tablero de seis fichas, cada uno con tres fichas de un mismo tipo, pero diferentes de su oponente. Consiste, por un lado, en lograr alinear las tres fichas formando una raya horizontal, vertical o diagonal, y por otro, en tratar de entorpecer los movimientos del contrario para evitar que el usuario o la máquina consigan alinear sus fichas antes.

\section{Teorías de Aprendizaje}

Entre las principales teorías que sustentan el hecho de que el juego de Tres en Raya estimula la cognición de los niños, podemos citar el estudio de Feuerstein, Klein, \& Tannenbaum (1991), en relación al desarrollo de habilidades cognitivas, quien considera que el juego sin lugar a dudas es un medio de aprendizaje que ocurre de forma vivencial. Sostiene que durante su ejecución se producen situaciones de percepción, atención, memoria y razonamiento, generando cambios en el pensamiento y el comportamiento. Estas capacidades que se producen en el cerebro se denominan modificabilidades cognitivas. Esta teoría pretende desarrollar la capacidad humana modificándola a través de la exposición directa a los estímulos y a la experiencia, a través del aprendizaje formal e informal, destacando el papel especial del mediador. Por tanto la mediación para que produzca modificabilidad cognitiva, ha de ser intencionada, con significado, que genere pensamiento positivo, 
que logre controlar y regular sus metas, animando a compartir, fomentando empatía pero al mismo tiempo consciente de la individualidad, causando en el niño desafío, confrontación, con respuestas divergentes para la solución de problemas.

En este mismo contexto, la perspectiva de Vygotsky (2000), radicaliza que el desarrollo cognitivo ocurre por la intervención de otra persona, siendo capaz de producirla a través de una buena mediación. Introduce el concepto, de Zona de Desarrollo Próximo (ZDP), que se define como la distancia que hay entre los resultados del aprendizaje autónomo del niño (nivel actual de desarrollo) y los resultados posibles con intervención pedagógica, entendida como la guía de un adulto o en colaboración con otro compañero más capaz (nivel de desarrollo potencial). Esta zona es diferente según la persona y, en este sentido, considerando esta diversidad, pretende que el alumnado alcance los mayores y mejores resultados posibles dentro de su ZDP.

Finalmente, Lipman (1998), basa su teoría en el lenguaje a través de técnicas de diálogo. Su postulado es muy cercano a la propuesta de Feuerstein, y resulta complementaria y potenciadora de la teoría de la modificabilidad cognitiva. El Diálogo estimula la reflexión, a través de preguntas y de respuestas elaboradas nuevamente en forma de pregunta, proceso que conduce al desarrollo de habilidades de razonamiento, clarifica significados, analiza conceptos, descubre supuestos implícitos, es decir creando un escenario intencionado para pensar bien y pensar autónomamente. Al asociar los supuestos teóricos de la modificabilidad cognitiva y el juego de Tres en Raya, se deduce que durante sus niveles de complejidad los niños expuestos a la vivencia del juego presumiblemente fortalecerán una serie de habilidades cognitivas, tales como la navegación espacial, el razonamiento, la memoria y la percepción tridimensional.

\section{Inteligencia Artificial para Videojuegos}

La Inteligencia Artificial (IA) es un campo de las ciencias computacionales que se puede aplicar en el proceso de enseñanza aprendizaje, que fomenta el razonamiento lógico, para resolver problemas complejos y encontrar soluciones de una manera más rápida y segura. La IA otorga a la computadora la capacidad de aparentar un raciocinio humano, y logra el dominio del aprendizaje por el reforzamiento y ejercitación, favorece procesos de construcción de conocimiento, reconoce una extensa gama de errores de razonamiento, provee conjuntos de problemas distintos y gradúa la dificultad relativa (Bello, 2002), (Rich \& Knight, 1994), (Torres Vinueza, Fuertes, Villacís Silva, Zambrano Rivera, \& Prócel Silva, 2013), (Villacis, Fuertes, Bustamante, Almachi, Procel, Fuertes, \& Toulkeridis, 2014). En este proyecto se ha seleccionado la combinación de técnicas heurísticas que hacen posible resolver más rápidamente problemas conocidos o similares a otros conocidos.

\section{Máquina de Estados Finitos}

Una máquina de estados finitos es un dispositivo o un modelo de un dispositivo, el cual tiene un número de estados que pueden ser activados en cualquier momento dado. Esta puede operar sobre entradas que toman cualquier transición desde un estado a otro, o pueden causar una salida o acción que puede tomar. Una máquina de estados finitos puede solamente estar en un estado en un determinado tiempo (Buckland, 2005).

\section{Series Numéricas}

Una serie numérica es la suma de una sucesión ordenada de elementos. Una serie es finita cuando se conoce el número de elementos o cuando el número de sumandos termina (De Burgos, 2013). En forma general una serie infinita se representa por $\sum_{n=1}^{\infty} u_{n}$ donde $u_{n}$ representa la ley de desarrollo y $\mathrm{n}=\{1,2,3,4, \ldots\}$, entonces:

$$
\sum_{n=1}^{\infty} u_{n}=u_{1}+u_{2}+u_{3}+\cdots+u_{n}+\cdots
$$

\section{DISEÑO E IMPLEMENTACIÓN}

En esta sección se muestra cómo se diseñó e implementó el videojuego didáctico “Tres en Raya”. El proyecto consistió de cuatro niveles de dificultad, un nivel para jugar entre dos usuarios y tres niveles para jugar contra el jugador controlado por la computadora (JCC) con IA. 


\section{Modelo IA, caso Tres en Raya}

Para el modelo de IA de la aplicación se han empleado técnicas heurísticas tanto débiles como fuertes, en el cual se utiliza un método numérico basado en series numéricas, que son representadas por listas enlazadas y arreglos. Estas se encargan de almacenar los diferentes movimientos hechos por la misma aplicación que viene a ser el jugador controlado por la computadora (JCC) y el usuario, donde cada movimiento se opera en base a una máquina de estados finitos. En la Tabla 1, se indica el estado inicial de todo el arreglo (i.e., cero) y corresponde a un espacio vacío o a un casillero libre:

\begin{tabular}{ll}
\hline Objeto & Estado \\
\hline Usuario & 1 \\
Jugador controlado por la computadora (JCC) & 3 \\
Espacio vacío & 0 \\
\hline
\end{tabular}

Tabla 1. Estados finitos del juego.

Fuente: Elaboración propia.

Así, dado:

$$
\begin{gathered}
V_{k} \in \mathbb{E} \text {, desde } k=0 \text {, hasta } k=8 \\
V_{k} \in \mathbb{E} \text {, para } 0 \leq k \leq 8
\end{gathered}
$$

El vector $V_{k}$ está representado en memoria RAM por un arreglo unidimensional llamado mArregloJugadas,

\begin{tabular}{|c|c|c|c|c|c|c|c|c|c|}
\hline \multirow[t]{2}{*}{ mArregloJugadas } & [0] & [1] & [2] & [3] & [4] & [5] & [6] & [7] & [8] \\
\hline & 0 & 0 & 0 & 0 & 0 & 0 & 0 & 0 & 0 \\
\hline
\end{tabular}
cuyos valores iniciales corresponden a cero, como se puede ver en la Figura 1:

Figura 1. Representación del vector $\mathrm{V}_{\mathrm{k}}$ a través de un arreglo unidimensional.

Fuente: Elaboración propia.

El método numérico basado en series finitas se indica en lo Cuadro 1, donde cada serie finita ha sido obtenida en base a una sumatoria que representa a un valor acumulado en una determinada fila, columna o diagonal del juego del Tres en Raya:

\begin{tabular}{|l|c|c|c|}
\hline Filas & $\sum_{i=0}^{n=2} f_{i}=a$ & $\sum_{i=3}^{n=5} f_{i}=b$ & $\sum_{i=6}^{n=8} f_{i}=c$ \\
\hline Columnas & $\sum_{\substack{i=0 \\
\text { step } 3}}^{n=6} c_{i}=d$ & $\sum_{\substack{i=1 \\
\text { Step } 3}}^{n=7} c_{i}=e$ & $\sum_{\substack{i=2 \\
\text { Step } 3}}^{n=8} c_{i}=f$ \\
\hline Diagonales & $\sum_{\substack{i=0 \\
n=8}}^{n=6} d_{i}=g$ & $\sum_{\substack{i=2 \\
\text { Step } 2}}^{n=6} d_{i}=h$ & \\
\hline Diagonales (Caso & $\sum^{n=8} d_{i}=x$ & $\sum_{\substack{i=2 \\
\text { Trivial) }}}^{n=6} d_{i}=y$ & \\
\hline
\end{tabular}

Cuadro 1. Método numérico basado en series finitas.

Fuente: Elaboración propia.

Caso 1: Bloquea el Jugador controlado por la computadora al usuario. En este caso de debe considerar lo siguiente:

$$
\text { si } a=2 \vee b=2 \vee c=2 \vee d=2 \vee e=2 \vee f=2 \vee g=2 \vee h=2
$$


Entonces se va a generar:

$$
\text { si } v_{[k]}=0 \rightarrow v_{[k]}:=3 \wedge \text { Bloquea JCC }
$$

A continuación en la Figura 2 se muestra un ejemplo para el Caso 1:

\begin{tabular}{|c|c|c|}
\hline $\mathrm{k}=0$ & $\mathrm{k}=1$ & $\mathrm{k}=2$ \\
$\mathrm{P}_{0}=0$ & $\mathrm{P}_{1}=0$ & $\mathrm{P}_{2}=0$ \\
\hline $\mathrm{k}=3$ & $\mathrm{k}=4$ & $\mathrm{k}=5$ \\
$\mathrm{X}$ & \\
$\mathrm{P}_{3}=0$ & $\mathrm{P}_{4}=1$ & $\mathrm{P}_{5}=0$ \\
\hline $\mathrm{k}=6$ & $\mathrm{k}=7$ & $\mathrm{k}=8$ \\
$\mathrm{P}_{6}=0$ & $\mathrm{P}_{7}=0$ & $\mathrm{P}_{8}=0$ \\
\hline
\end{tabular}

\begin{tabular}{|c|c|c|}
\hline \multicolumn{3}{|c|}{ Segunda Jugada } \\
\hline $\begin{array}{c}\mathrm{k}=0 \\
0\end{array}$ & $k=1$ & $k=2$ \\
\hline$P_{0}=3$ & $P_{1}=0$ & $P_{2}=0$ \\
\hline$k=3$ & $\begin{array}{c}k=4 \\
\mathbf{X}\end{array}$ & $k=5$ \\
\hline$P_{3}=0$ & $P_{4}=1$ & $P_{5}=0$ \\
\hline $\mathrm{k}=6$ & $\mathrm{k}=7$ & $\mathrm{k}=8$ \\
\hline$P_{6}=0$ & $\mathrm{P}_{7}=0$ & $P_{8}=0$ \\
\hline
\end{tabular}

\begin{tabular}{|c|c|c|}
\hline \multicolumn{3}{|c|}{ Tercera Jugada } \\
\hline $\begin{array}{c}\mathrm{k}=0 \\
0 \\
\mathrm{P}_{0}=3\end{array}$ & $\begin{array}{l}\mathrm{k}=1 \\
\mathrm{P}_{1}=0\end{array}$ & $\begin{array}{c}\mathrm{k}=2 \\
\mathrm{X} \\
\mathrm{P}_{2}=1\end{array}$ \\
\hline $\begin{aligned} k & =3 \\
P_{3} & =0\end{aligned}$ & $\begin{array}{c}\mathrm{k}=4 \\
\mathrm{X} \\
\mathrm{P}_{4}=1\end{array}$ & $\begin{aligned} k & =5 \\
P_{5} & =0\end{aligned}$ \\
\hline$k=6$ & $k=7$ & $k=8$ \\
\hline$P_{6}=0$ & $P_{7}=0$ & $P_{8}=0$ \\
\hline
\end{tabular}

$$
\sum_{\substack{i=2 \\ \text { Step } 2}}^{n=6} d_{i}=2
$$

\begin{tabular}{|c|c|c|}
\multicolumn{3}{c}{ Cuarta Jugada } \\
\hline $\begin{array}{c}\mathrm{k}=0 \\
0\end{array}$ & $\mathrm{k}=1$ & $\mathrm{k}=2$ \\
$\mathrm{P}$ & $\mathrm{X}$ \\
$\mathrm{K}=3$ & $\mathrm{P}_{1}=0$ & $\mathrm{P}_{2}=1$ \\
\hline & $\mathrm{k}=4$ & $\mathrm{~K}=5$ \\
$\mathrm{P}_{3}=0$ & $\mathrm{P}_{4}=1$ & $\mathrm{P}_{5}=0$ \\
\hline $\mathrm{K}=6$ & $\mathrm{k}=7$ & $\mathrm{~K}=8$ \\
0 & & \\
$\mathrm{P}_{6}=3$ & $\mathrm{P}_{7}=0$ & $\mathrm{P}_{8}=0$ \\
\hline
\end{tabular}

El jugador controlado por la computadora (JCC) bloquea al

\begin{tabular}{|c|c|c|}
\hline \multicolumn{3}{|c|}{ Séptima Jugada } \\
\hline $\begin{array}{c}\mathrm{k}=0 \\
0 \\
\mathrm{P}_{0}=3\end{array}$ & $\begin{array}{c}\mathrm{k}=1 \\
\mathrm{X} \\
\mathrm{P}_{1}=1\end{array}$ & $\begin{array}{c}\mathrm{k}=2 \\
\mathrm{X} \\
\mathrm{P}_{2}=1\end{array}$ \\
\hline $\begin{array}{c}k=3 \\
X \\
P_{3}=1\end{array}$ & $\begin{array}{c}\mathrm{k}=4 \\
\mathrm{X} \\
\mathrm{P}_{4}=1\end{array}$ & $\begin{array}{c}\mathrm{k}=5 \\
\mathbf{0} \\
\mathrm{P}_{5}=3\end{array}$ \\
\hline $\begin{array}{c}\mathrm{k}=6 \\
0 \\
\mathrm{P}_{6}=3\end{array}$ & $k=7$ & $k=8$ \\
\hline
\end{tabular}
usuario

\begin{tabular}{|c|c|c|}
\hline \multicolumn{3}{|c|}{ Quinta Jugada } \\
\hline $\begin{array}{c}\mathrm{k}=0 \\
\mathbf{0} \\
\mathrm{P}_{0}=3\end{array}$ & $\begin{aligned} \mathrm{k} & =1 \\
\mathrm{P}_{1} & =0\end{aligned}$ & $\begin{array}{c}\mathrm{k}=2 \\
\mathrm{X} \\
\mathrm{P}_{2}=1\end{array}$ \\
\hline $\begin{array}{c}\mathrm{K}=3 \\
\mathrm{X} \\
\mathrm{P}_{3}=1\end{array}$ & $\begin{array}{c}\mathrm{K}=4 \\
\mathrm{X} \\
\mathrm{P}_{4}=1\end{array}$ & $\begin{array}{l}\mathrm{k}=5 \\
\mathrm{P}_{5}=0\end{array}$ \\
\hline $\begin{array}{c}k=6 \\
0 \\
P_{8}=3\end{array}$ & $\begin{aligned} \mathrm{k} & =7 \\
\mathrm{P}_{7} & =0\end{aligned}$ & $\begin{aligned} k & =8 \\
P_{8} & =0\end{aligned}$ \\
\hline
\end{tabular}

$$
\sum_{\substack{i=1 \\ \text { Step } 3}}^{n=7} c_{i}=2
$$

$$
\sum_{i=3}^{n=5} f_{i}=2
$$

\begin{tabular}{|c|c|c|}
\hline $\mathrm{k}=0$ & $\mathrm{k}=1$ & $\mathrm{k}=2$ \\
0 & $\mathrm{X}$ & $\mathrm{X}$ \\
$\mathrm{P}_{0}=3$ & $\mathrm{P}_{1}=1$ & $\mathrm{P}_{2}=1$ \\
\hline $\mathrm{k}=3$ & $\mathrm{k}=4$ & $\mathrm{k}=5$ \\
$\mathrm{X}$ & $\mathrm{X}$ & 0 \\
$\mathrm{P}_{3}=1$ & $\mathrm{P}_{4}=1$ & $\mathrm{P}_{5}=3$ \\
\hline $\mathrm{k}=6$ & $\mathrm{k}=7$ & $\mathrm{k}=8$ \\
0 & 0 & \\
$\mathrm{P}_{6}=3$ & $\mathrm{P}_{7}=3$ & $\mathrm{P}_{8}=0$ \\
\hline
\end{tabular}

El jugador controlado por la computadora (JCC) bloquea al usuario

\begin{tabular}{|c|c|c|} 
Sexta Jugada \\
\begin{tabular}{|c|c|c|}
$\mathrm{k}=0$ & $\mathrm{k}=1$ & $\mathrm{k}=2$ \\
0 & & $\mathrm{X}$ \\
$\mathrm{P}_{0}=3$ & $\mathrm{P}_{1}=0$ & $\mathrm{P}_{2}=1$ \\
\hline $\mathrm{k}=3$ & $\mathrm{k}=4$ & $\mathrm{k}=5$ \\
$\mathrm{X}$ & $\mathrm{X}$ & 0 \\
$\mathrm{P}_{3}=1$ & $\mathrm{P}_{4}=1$ & $\mathrm{P}_{5}=3$ \\
\hline $\mathrm{k}=6$ & $\mathrm{k}=7$ & $\mathrm{k}=8$ \\
0 & & \\
$\mathrm{P}_{8}=3$ & $\mathrm{P}_{7}=0$ & $\mathrm{P}_{8}=0$ \\
\hline
\end{tabular}
\end{tabular}

El jugador controlado por la computadora (ICC) bloquea al usuario

\begin{tabular}{|c|c|c|}
\hline $\mathrm{k}=0$ & $\mathrm{k}=1$ & $\mathrm{k}=2$ \\
0 & $\mathrm{X}$ & $\mathrm{X}$ \\
$\mathrm{P}_{0}=3$ & $\mathrm{P}_{1}=1$ & $\mathrm{P}_{2}=1$ \\
\hline $\mathrm{k}=3$ & $\mathrm{k}=4$ & $\mathrm{k}=5$ \\
$\mathrm{X}$ & $\mathrm{X}$ & 0 \\
$\mathrm{P}_{3}=1$ & $\mathrm{P}_{4}=1$ & $\mathrm{P}_{5}=3$ \\
\hline $\mathrm{k}=6$ & $\mathrm{k}=7$ & $\mathrm{k}=8$ \\
0 & 0 & $\mathrm{X}$ \\
$\mathrm{P}_{6}=3$ & $\mathrm{P}_{7}=3$ & $\mathrm{P}_{8}=1$ \\
\hline
\end{tabular}

Se produce un empate entre el usuario y el jugador controlado por la computadora (JCC)

mArregloJ ugadas
\begin{tabular}{|c|c|c|c|c|c|c|c|c|}
{$[0]$} & {$[1]$} & {$[2]$} & {$[3]$} & {$[4]$} & {$[5]$} & {$[6]$} & {$[7]$} & {$[8]$} \\
\hline 3 & 1 & 1 & 1 & 1 & 3 & 3 & 3 & 1 \\
\hline
\end{tabular}

Figura 2. Ejemplo para el Caso 1.

Fuente: Elaboración propia.

Caso 2: Gana el Jugador controlado por la computadora (JCC) al usuario. En este caso de debe considerar lo siguiente:

$$
\text { si } a=6 \vee b=6 \vee c=6 \vee d=6 \vee e=6 \vee f=6 \vee g=6 \vee h=6
$$


Entonces se obtendrá:

$$
\text { si } v_{[k]}=0 \rightarrow v_{[k]}:=3 \wedge \text { Gana JCC }
$$

A continuación en la Figura 3 se muestra un ejemplo para el Caso 2:

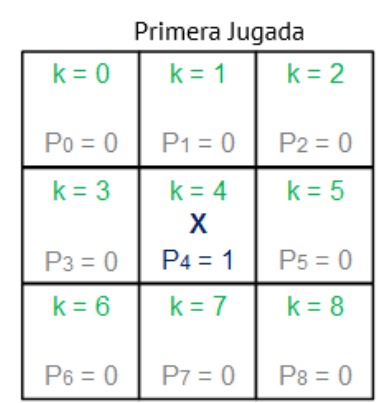

Segunda Jugada
\begin{tabular}{|c|c|c|}
\hline $\mathrm{k}=0$ & $\mathrm{k}=1$ & $\mathrm{k}=2$ \\
& & 0 \\
$\mathrm{P}_{0}=0$ & $\mathrm{P}_{1}=0$ & $\mathrm{P}_{2}=3$ \\
\hline $\mathrm{k}=3$ & $\mathrm{k}=4$ & $\mathrm{k}=5$ \\
$\mathrm{X}$ & \\
$\mathrm{P}_{3}=0$ & $\mathrm{P}_{4}=1$ & $\mathrm{P}_{5}=0$ \\
\hline $\mathrm{k}=6$ & $\mathrm{k}=7$ & $\mathrm{k}=8$ \\
$\mathrm{P}_{6}=0$ & $\mathrm{P}_{7}=0$ & $\mathrm{P}_{8}=0$ \\
\hline
\end{tabular}

\begin{tabular}{|c|c|c|}
\hline \multicolumn{3}{|c|}{ Cuarta Jugada } \\
\hline $\begin{array}{c}\mathrm{k}=0 \\
\mathrm{X} \\
\mathrm{P}_{0}=1\end{array}$ & $\begin{array}{c}\mathrm{k}=1 \\
\mathrm{P}_{1}=0\end{array}$ & $\begin{array}{c}\mathrm{k}=2 \\
0 \\
\mathrm{P}_{2}=3\end{array}$ \\
\hline $\begin{aligned} \mathrm{k} & =3 \\
\mathrm{P}_{3} & =0\end{aligned}$ & $\begin{array}{c}k=4 \\
X \\
P_{4}=1\end{array}$ & $\begin{aligned} k & =5 \\
P_{5} & =0\end{aligned}$ \\
\hline $\begin{array}{l}\mathrm{k}=6 \\
\mathrm{P}_{6}=0\end{array}$ & $\begin{array}{c}\mathrm{k}=7 \\
\mathrm{P}_{7}=0\end{array}$ & $\begin{array}{c}\mathrm{k}=8 \\
0 \\
\mathrm{P}_{8}=3\end{array}$ \\
\hline
\end{tabular}

El jugador controlado por la computadora (JCC) bloquea al usuario

\begin{tabular}{|c|c|c|}
\hline $\begin{array}{c}\mathrm{k}=0 \\
\mathrm{X}\end{array}$ & $\mathrm{k}=1$ & $\mathrm{k}=2$ \\
$\mathrm{P}_{0}=1$ & $\mathrm{P}_{1}=0$ & $\mathrm{P}_{2}=3$ \\
\hline $\mathrm{k}=3$ & $\mathrm{k}=4$ & $\mathrm{k}=5$ \\
& $\mathrm{X}$ & \\
$\mathrm{P}_{3}=0$ & $\mathrm{P}_{4}=1$ & $\mathrm{P}_{5}=0$ \\
\hline $\mathrm{k}=6$ & $\mathrm{k}=7$ & $\mathrm{k}=8$ \\
$\mathrm{X}$ & & 0 \\
$\mathrm{P}_{6}=1$ & $\mathrm{P}_{7}=0$ & $\mathrm{P}_{8}=3$ \\
\hline
\end{tabular}

Tercera Jugada
\begin{tabular}{|c|c|c|}
\hline $\begin{array}{c}\mathrm{k}=0 \\
\mathbf{X}\end{array}$ & $\mathrm{k}=1$ & $\mathrm{k}=2$ \\
$\mathrm{P}_{0}=1$ & $\mathrm{P}_{1}=0$ & $\mathrm{P}_{2}=3$ \\
\hline $\mathrm{k}=3$ & $\mathrm{k}=4$ & $\mathrm{k}=5$ \\
& $\mathbf{X}$ & \\
$\mathrm{P}_{3}=0$ & $\mathrm{P}_{4}=1$ & $\mathrm{P}_{5}=0$ \\
\hline $\mathrm{k}=6$ & $\mathrm{k}=7$ & $\mathrm{k}=8$ \\
$\mathrm{P}_{6}=0$ & $\mathrm{P}_{7}=0$ & $\mathrm{P}_{8}=0$ \\
\hline
\end{tabular}

$$
\sum_{\substack{i=0 \\ \text { Step } 4}}^{n=8} d_{i}=2
$$

$$
\sum_{\substack{i=2 \\ \text { Step } 3}}^{n=8} c_{i}=6
$$

Sexta Jugada
\begin{tabular}{|c|c|c|}
\hline $\mathrm{k}=0$ & $\mathrm{k}=1$ & $\mathrm{k}=2$ \\
$\mathbf{X}$ & & $\mathbf{0}$ \\
$\mathrm{P}_{0}=1$ & $\mathrm{P}_{1}=0$ & $\mathrm{P}_{2}=3$ \\
\hline $\mathrm{k}=3$ & $\mathrm{k}=4$ & $\mathrm{k}=5$ \\
& $\mathbf{X}$ & $\mathbf{0}$ \\
$\mathrm{P}_{3}=0$ & $\mathrm{P}_{4}=1$ & $\mathrm{P}_{5}=3$ \\
\hline $\mathrm{k}=6$ & $\mathrm{k}=7$ & $\mathrm{k}=8$ \\
$\mathbf{X}$ & & $\mathbf{0}$ \\
$\mathrm{P}_{6}=1$ & $\mathrm{P}_{7}=0$ & $\mathrm{P}_{8}=3$ \\
\hline
\end{tabular}

Gana el jugador controlado por la computadora (JCC)

\begin{tabular}{cc|c|c|c|c|c|c|c|c|} 
mArregloJugadas & {$[0]$} & {$[1]$} & {$[2]$} & {$[3]$} & {$[4]$} & {$[5]$} & {$[6]$} & {$[7]$} & {$[8]$} \\
\hline 1 & 0 & 3 & 0 & 1 & 3 & 1 & 0 & 3 \\
\hline
\end{tabular}

Figura 3. Ejemplo para el Caso 2.

Fuente: Elaboración propia.

Caso Trivial: Bloqueo en las diagonales. En este caso trivial de debe considerar lo siguiente:

$$
\text { si } x=5 \vee y=5
$$

Este caso trivial genera:

$$
\text { si } v_{[k]}=0 \rightarrow v_{[k]}:=3 \wedge \text { Bloquea JCC }
$$


A continuación en la Figura 4 se muestra un ejemplo para el Caso Trivial:

Primera Jugada
\begin{tabular}{|c|c|c|}
\hline $\mathrm{k}=0$ & $\mathrm{k}=1$ & $\mathrm{k}=2$ \\
$\mathrm{P}_{0}=0$ & $\mathrm{P}_{1}=0$ & $\mathrm{P}_{2}=0$ \\
\hline $\mathrm{k}=3$ & $\mathrm{k}=4$ & $\mathrm{k}=5$ \\
$\mathrm{X}$ & \\
$\mathrm{P}_{3}=0$ & $\mathrm{P}_{4}=1$ & $\mathrm{P}_{5}=0$ \\
\hline $\mathrm{k}=6$ & $\mathrm{k}=7$ & $\mathrm{k}=8$ \\
$\mathrm{P}_{8}=0$ & $\mathrm{P}_{7}=0$ & $\mathrm{P}_{8}=0$ \\
\hline
\end{tabular}

Cuarta Jugada

\begin{tabular}{|c|c|c|}
\hline $\begin{array}{c}\mathrm{k}=0 \\
\mathrm{X}\end{array}$ & $\mathrm{k}=1$ & $\mathrm{k}=2$ \\
$\mathrm{P}_{0}=1$ & $\mathrm{P}_{1}=0$ & $\mathrm{P}_{2}=3$ \\
\hline $\mathrm{k}=3$ & $\mathrm{k}=4$ & $\mathrm{k}=5$ \\
& $\mathrm{X}$ & \\
$\mathrm{P}_{3}=0$ & $\mathrm{P}_{4}=1$ & $\mathrm{P}_{5}=0$ \\
\hline $\mathrm{k}=6$ & $\mathrm{k}=7$ & $\mathrm{k}=8$ \\
& & 0 \\
$\mathrm{P}_{8}=0$ & $\mathrm{P}_{7}=0$ & $\mathrm{P}_{8}=3$ \\
\hline
\end{tabular}

Como primera prioridad, el jugador controlado por la computadora (JCC), coloca su ficha en una esquina

Séptima Jugada

\begin{tabular}{|c|c|c|}
\hline $\begin{array}{c}\mathrm{k}=0 \\
\mathrm{X}\end{array}$ & $\mathrm{k}=1$ & $\mathrm{k}=2$ \\
$\mathrm{P}_{0}=1$ & $\mathrm{P}_{1}=0$ & $\mathrm{P}_{2}=3$ \\
\hline $\mathrm{k}=3$ & $\mathrm{k}=4$ & $\mathrm{k}=5$ \\
0 & $\mathrm{X}$ & $\mathrm{X}$ \\
$\mathrm{P}_{3}=3$ & $\mathrm{P}_{4}=1$ & $\mathrm{P}_{5}=1$ \\
\hline $\mathrm{k}=6$ & $\mathrm{k}=7$ & $\mathrm{k}=8$ \\
$\mathrm{X}$ & & 0 \\
$\mathrm{P}_{6}=1$ & $\mathrm{P}_{7}=0$ & $\mathrm{P}_{8}=3$ \\
\hline
\end{tabular}

$$
\sum_{\substack{i=2 \\ \text { Step } 2}}^{n=6} d_{i}=5
$$

\section{Segunda Jugada}

\begin{tabular}{|c|c|c|}
\hline$k=0$ & $k=1$ & $k=2$ \\
$P_{0}=0$ & $P_{1}=0$ & $P_{2}=0$ \\
\hline$k=3$ & $k=4$ & $k=5$ \\
$X$ & \\
$P_{3}=0$ & $P_{4}=1$ & $P_{5}=0$ \\
\hline$k=6$ & $k=7$ & $k=8$ \\
& & 0 \\
$P_{8}=0$ & $P_{7}=0$ & $P_{8}=3$ \\
\hline
\end{tabular}

Quinta Jugada

\begin{tabular}{|c|c|c|}
\hline $\begin{array}{c}\mathrm{k}=0 \\
\mathrm{X}\end{array}$ & $\mathrm{k}=1$ & $\mathrm{k}=2$ \\
$\mathrm{P}_{0}=1$ & $\mathrm{P}_{1}=0$ & $\mathrm{P}_{2}=3$ \\
\hline $\mathrm{k}=3$ & $\mathrm{k}=4$ & $\mathrm{k}=5$ \\
& $\mathrm{X}$ & $\mathrm{X}$ \\
$\mathrm{P}_{3}=0$ & $\mathrm{P}_{4}=1$ & $\mathrm{P}_{5}=1$ \\
\hline $\mathrm{k}=6$ & $\mathrm{k}=7$ & $\mathrm{k}=8$ \\
& & 0 \\
$\mathrm{P}_{8}=0$ & $\mathrm{P}_{7}=0$ & $\mathrm{P}_{8}=3$ \\
\hline
\end{tabular}

$$
\sum_{\substack{i=1 \\ \text { Step } 3}}^{n=7} c_{i}=2
$$

Octava Jugada

\begin{tabular}{|c|c|c|}
\hline $\begin{array}{c}\mathrm{k}=0 \\
\mathrm{X}\end{array}$ & $\mathrm{k}=1$ & $\mathrm{k}=2$ \\
$\mathrm{P}_{0}=1$ & $\mathrm{P}_{1}=0$ & $\mathrm{P}_{2}=3$ \\
\hline $\mathrm{k}=3$ & $\mathrm{k}=4$ & $\mathrm{k}=5$ \\
0 & $\mathrm{X}$ & $\mathrm{X}$ \\
$\mathrm{P}_{3}=3$ & $\mathrm{P}_{4}=1$ & $\mathrm{P}_{5}=1$ \\
\hline $\mathrm{k}=6$ & $\mathrm{k}=7$ & $\mathrm{k}=8$ \\
$\mathrm{X}$ & 0 & 0 \\
$\mathrm{P}_{8}=1$ & $\mathrm{P}_{7}=3$ & $\mathrm{P}_{8}=3$ \\
\hline
\end{tabular}

Como segunda prioridad, el jugador controlado por la computadora (JCC), coloca su ficha en un casillero libre de forma aleatoria
Tercera Jugada

\begin{tabular}{|c|c|c|}
\hline $\begin{array}{c}\mathrm{k}=0 \\
\mathrm{X} \\
\mathrm{P}_{0}=1\end{array}$ & $\mathrm{k}=1$ & $\mathrm{k}=2$ \\
\hline $\mathrm{k}=3$ & $\mathrm{P}=4$ & $\mathrm{P}_{2}=0$ \\
& $\mathrm{X}$ & $\mathrm{k}=5$ \\
$\mathrm{P}_{3}=0$ & $\mathrm{P}_{4}=1$ & $\mathrm{P}_{5}=0$ \\
\hline $\mathrm{k}=6$ & $\mathrm{k}=7$ & $\mathrm{k}=8$ \\
& & 0 \\
$\mathrm{P}_{8}=0$ & $\mathrm{P}_{7}=0$ & $\mathrm{P}_{8}=3$ \\
\hline
\end{tabular}

$$
\sum_{\substack{i=0 \\ \text { Step } 4}}^{n=8} d_{i}=5
$$

Sexta Jugada

\begin{tabular}{|c|c|c|}
\hline $\begin{array}{c}\mathrm{k}=0 \\
\mathrm{X}\end{array}$ & $\mathrm{k}=1$ & $\mathrm{k}=2$ \\
$\mathrm{P}_{0}=1$ & $\mathrm{P}_{1}=0$ & $\mathrm{P}_{2}=3$ \\
\hline $\mathrm{k}=3$ & $\mathrm{k}=4$ & $\mathrm{k}=5$ \\
0 & $\mathrm{X}$ & $\mathrm{X}$ \\
$\mathrm{P}_{3}=3$ & $\mathrm{P}_{4}=1$ & $\mathrm{P}_{5}=1$ \\
\hline $\mathrm{k}=6$ & $\mathrm{k}=7$ & $\mathrm{k}=8$ \\
& & 0 \\
$\mathrm{P}_{8}=0$ & $\mathrm{P}_{7}=0$ & $\mathrm{P}_{8}=3$ \\
\hline
\end{tabular}

El jugador controlado por la computadora (JCC) bloquea al usuario

Novena Jugada

\begin{tabular}{|c|c|c|}
\hline $\mathrm{k}=0$ & $\mathrm{k}=1$ & $\mathrm{k}=2$ \\
$\mathrm{X}$ & $\mathrm{X}$ & 0 \\
$\mathrm{P}_{0}=1$ & $\mathrm{P}_{1}=1$ & $\mathrm{P}_{2}=3$ \\
\hline $\mathrm{k}=3$ & $\mathrm{k}=4$ & $\mathrm{k}=5$ \\
0 & $\mathrm{X}$ & $\mathrm{X}$ \\
$\mathrm{P}_{3}=3$ & $\mathrm{P}_{4}=1$ & $\mathrm{P}_{5}=1$ \\
\hline $\mathrm{k}=6$ & $\mathrm{k}=7$ & $\mathrm{k}=8$ \\
$\mathrm{X}$ & 0 & 0 \\
$\mathrm{P}_{6}=1$ & $\mathrm{P}_{7}=3$ & $\mathrm{P}_{8}=3$ \\
\hline
\end{tabular}

Se produce un empate entre el usuario y el jugador controlado por la computadora (JCC)

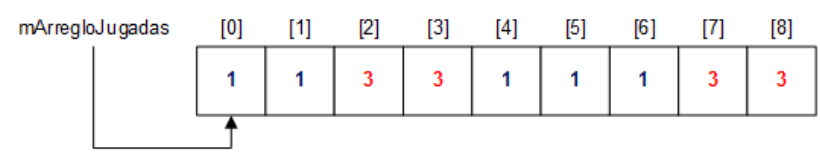

Figura 4. Ejemplo para el Caso Trivial.

Fuente: Elaboración propia.

\section{Especificación de Requerimientos}

El sistema tuvo que cumplir las siguientes tareas y roles: (1) Dos jugadores; (2) nivel principiante; (3) nivel intermedio; (4) nivel avanzado; (5) opción archivo; (6) opción ayuda; (7) opción ver. Las Figuras 5 y 6, muestran los diagramas de casos de uso de la aplicación del Juego del Tres en Raya y el diagrama de secuencias de ¿cómo cargar el juego?: 


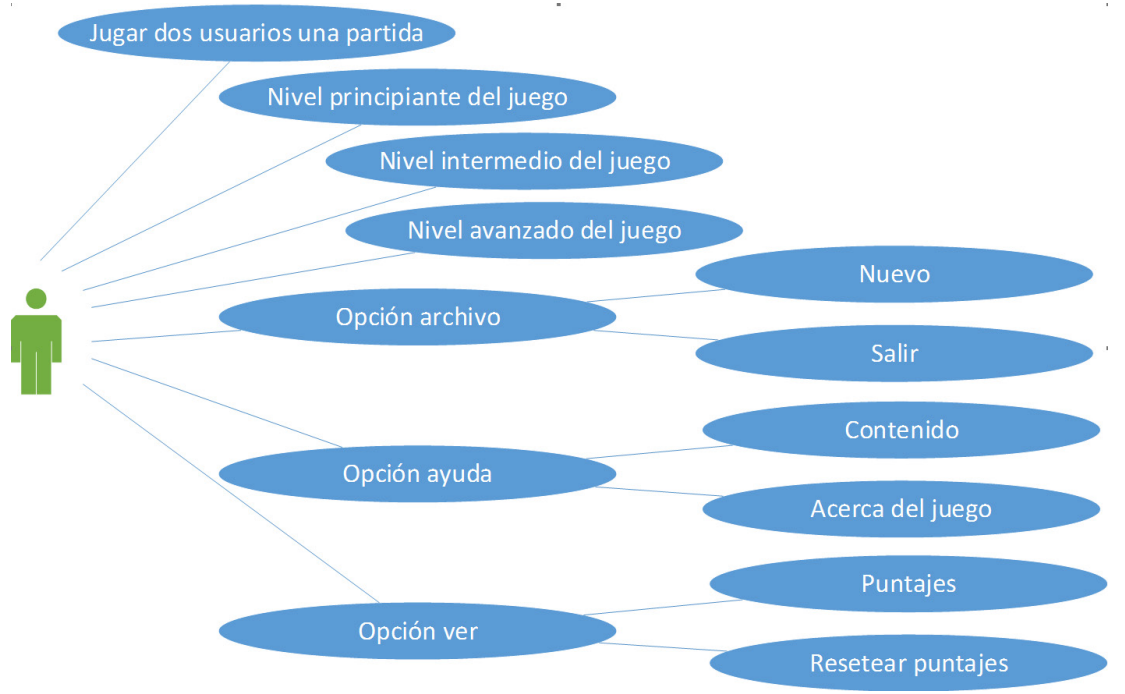

Figura 5. Caso de uso del Juego del Tres en Raya.

Fuente: Elaboración propia.

\section{Diseño Conceptual}

En esta fase, se diseñaron e implementaron archivos planos para la manipulación y almacenamiento de la información, como son puntajes, datos referenciales, configuraciones y usuarios del sistema. Así mismo, se determinó que la arquitectura a utilizar sería Cliente Servidor de 2-Capas, el cual se complementa con OOHDM, separando el diseño de interfaz con las reglas del negocio y los controladores del juego. El sistema posee cuatro controladores del juego los cuales son: (1) controlador de dos usuarios; (2) controlador de jugadas aleatorias, (3) controlador de jugadas inteligentes débiles, y (4) controlador de jugadas inteligentes fuertes. Además posee tres clases para el manejo de animaciones, manejo del sonido y el manejo de archivos planos del sistema.

\section{Modelo Navegacional}

En esta fase, el desarrollo de las interfaces estuvo marcado por el uso de formularios (los controles Form), los que permiten una adecuada forma para desarrollar vistas, además de ser estéticamente acertadas. Los objetos Navegacionales son: formulario del juego del Tres en Raya, formulario acerca del juego, formulario del contenido del juego y formulario de los puntajes del juego. Los contextos navegacionales son: seleccionar nivel de dificultad del juego, seleccionar las piezas del tablero del juego, menú archivo, menú ayuda del juego, menú ver puntajes.

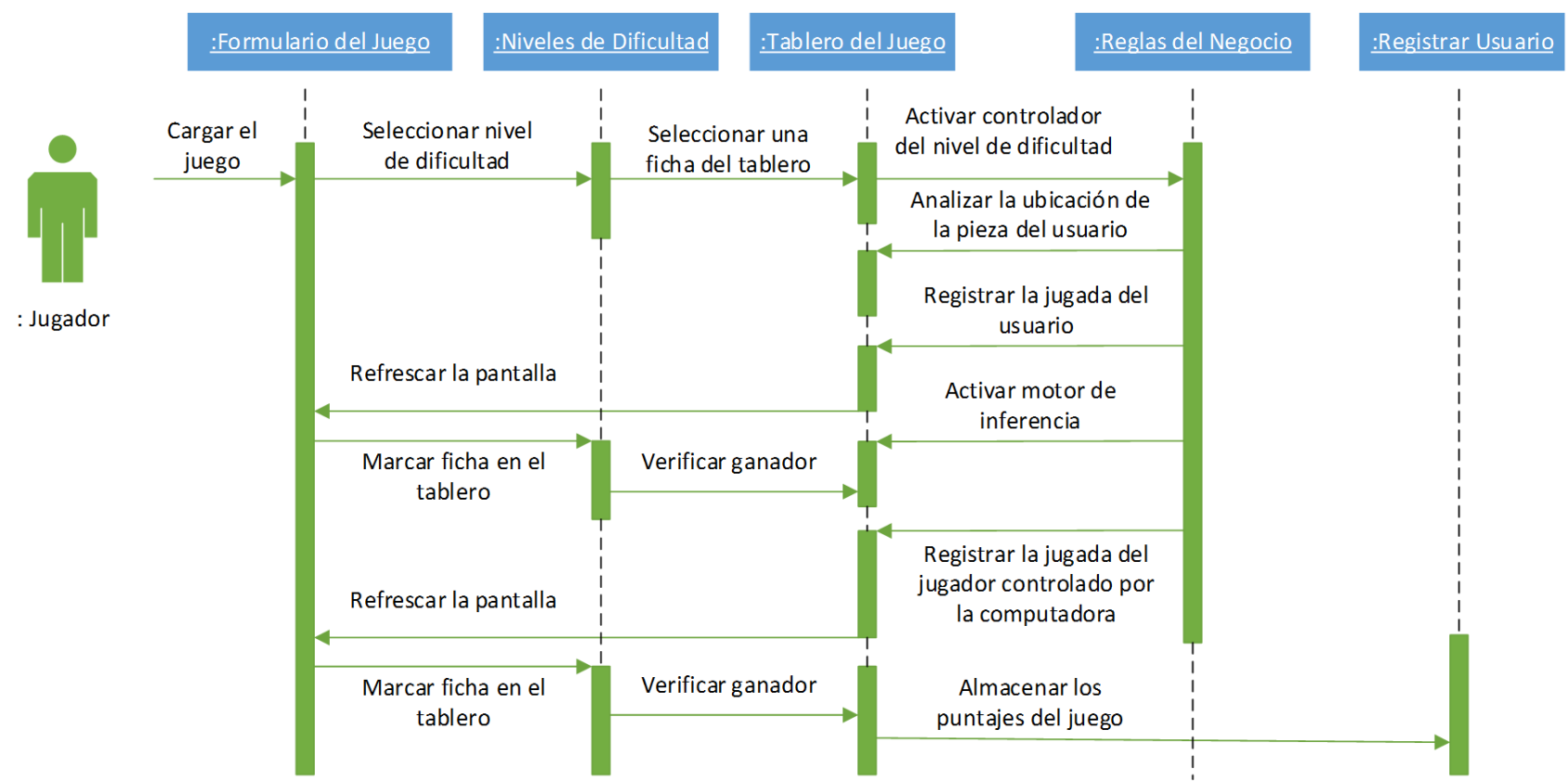

Figura 6. Diagrama de secuencias "Cargar el juego".

Fuente: Elaboración propia. 


\section{Construcción de la Interfaz Gráfica del Juego del Tres en Raya}

La interfaz del juego se divide en la clase Programa, que se encarga de mostrar la ventana principal de la aplicación representada por la clase frmTresEnRaya, que presenta los tres niveles de dificultad del juego (nivel principiante, nivel intermedio y nivel avanzado), y que contiene la opción de jugar dos usuarios entre sí. El juego además tiene tres formularios adicionales los cuales son: a) Formulario Acerca del juego (frmAcercaDe) que presenta la información de los creadores del juego sin fines de lucro y donde se indica el uso del logotipo animado de Pocoyo $^{1}$; b) Formulario Contenido (frmContenido) que presenta la información del contenido y funcionamiento del juego desarrollado en la herramienta de autor Articulate Studio; c) Formulario Puntajes (frmPuntajes) que presenta la información acerca de los puntajes obtenido por el usuario del juego. El juego del Tres en Raya tiene derechos reservados en la empresa Virtual Learning Solutions. La Figura 7 muestra la interfaz gráfica del videojuego didáctico y su ejecución en los tres niveles de dificultad:

[a]

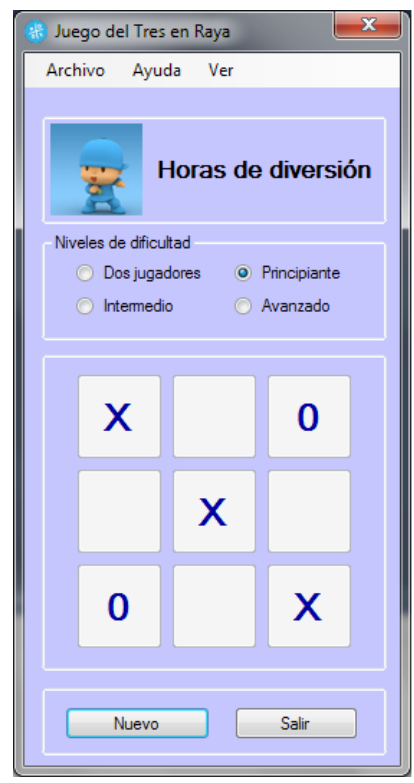

[b]

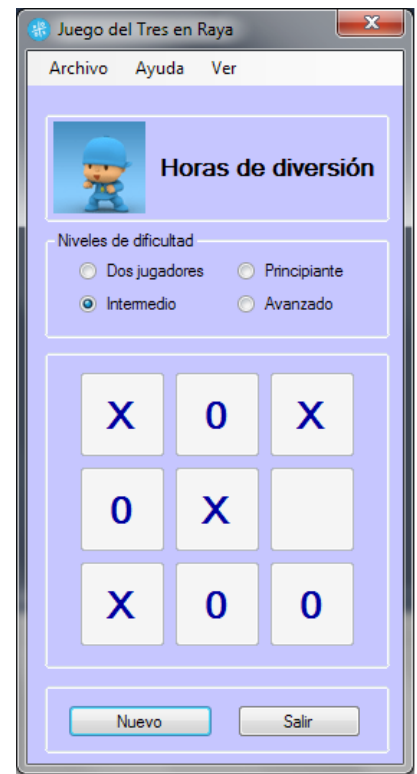

[c]

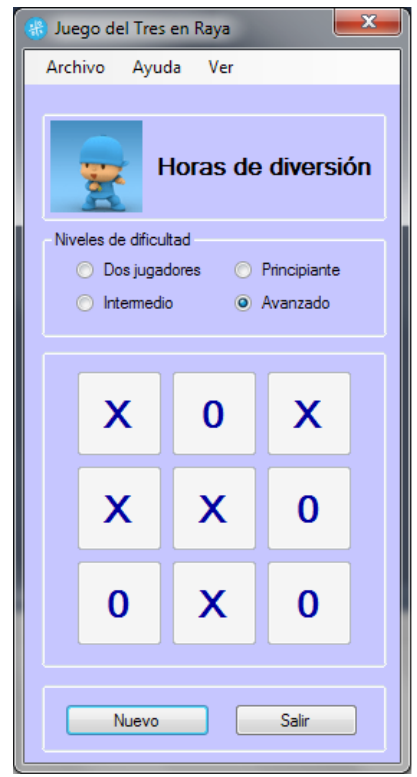

Figura 7. (a) Nivel principiante. (b) Nivel intermedio. (c) Nivel avanzado.

Fuente: Elaboración propia.

\section{EVALUACIÓN DE RESULTADOS}

Para la evaluación del juego de Tres en Raya, se receptó una muestra aleatoria a 30 niños/as comprendidos entre las edades de 7 a 11 años de una institución educativa, con la finalidad de conocer el nivel de aceptación del juego, como un generador desafiante al proceso mental cognitivo de los aprendizajes lúdicos. Mediante el juego que está enfocado a la creatividad, al razonamiento lógico; el desafío es ganarle al juego, el mismo que consta de tres niveles principiante, intermedio y avanzado. Para esto se aplicó un instrumento de medición estadístico de observación directa en varias computadoras, en un mismo tiempo determinado. Luego de su procesamiento estadístico se obtuvo los siguientes resultados:

En relación a la media aritmética, el 47.9\% gana al juego en el nivel 1, mientras que el 77.2 \% lo hace en el nivel 2. Esto se da cuando el niño/a ya está más familiarizado con el mismo. Sin embargo el $0 \%$ no logra ganar en el último nivel. Esto debido a que el grado de dificultad es mayor y por lo tanto tiene que desarrollar un mayor número de destrezas cognitivas y fisiológicas (concentración). En relación con la desviación estándar se presenta un fenómeno casi parecido, es decir; el nivel 1 tiene: 8.424, el dos: 9.326 y el tercero se ha desestimado, debido que en el nivel 1 nadie pierde, mientras que en el tercero nadie puede lograr su objetivo (i.e. ganarle al juego Tres en Raya.

A continuación nos apoyaremos también de manera experimental y teórica en el análisis de los gráficos siguientes en relación a los niveles de dificultad: El Gráfico 1(a) muestra que el 80\% de los/as niños /as comprendidos/as entre las edades de 7 a 9 años logran ganarle al juego Tres en Raya en el primer nivel, mientras

\footnotetext{
${ }^{1}$ Images @ Granada/Zinkia, 2015. Pocoyo @ Zinkia S. L. Bajo Licencia de G.V. La Serie, los logotipos y los personajes de la Serie Pocoyó son marcas registradas de Zinkia entertainment S. L. y se utilizan bajo licencia.
} 
que el 6.7\% entre las edades de 10 a11 años logran hacerlo. Esto indica que mientras menor edad tiene el/a niño/a, posee mayor interés por los juegos educativos, por lo que su aprendizaje se desarrolla de manera significativa.

El Gráfico 1(b) muestra que el 36.7\% de los/as niños/as comprendidos entre las edades de 7 a 10 años logran ganarle y también empatan con el juego, en cambio que sólo el 10.0\% logran ganar el juego. Es decir se observa que el 53,3\% no logran su objetivo de alcanzar el siguiente nivel. Esto constituye un indicador objetivo, que indica que el juego presenta mayor grado de dificultad, por lo que los/as niños/as tendrán que desarrollar nuevas destrezas cognitivas, haciéndole al juego más desafiante e interesante para el/a niño/a.

El Gráfico 1(c) muestra que el 0\% de los/as niños no alcanzan su objetivo de ganarle en este nivel al juego. Se observa que el 53,3\% entre las edades de 7-9 años solo logran empatar, más bien el 36.7\% pierden. Se observa además claramente que los /as niños/as entre los de 10 y 11 años, logran empatar en un 10\%. Por tanto cuando se incrementa el nivel del juego, este genera mayor dificultad para quienes desean alcanzar la meta de ganarle al juego.
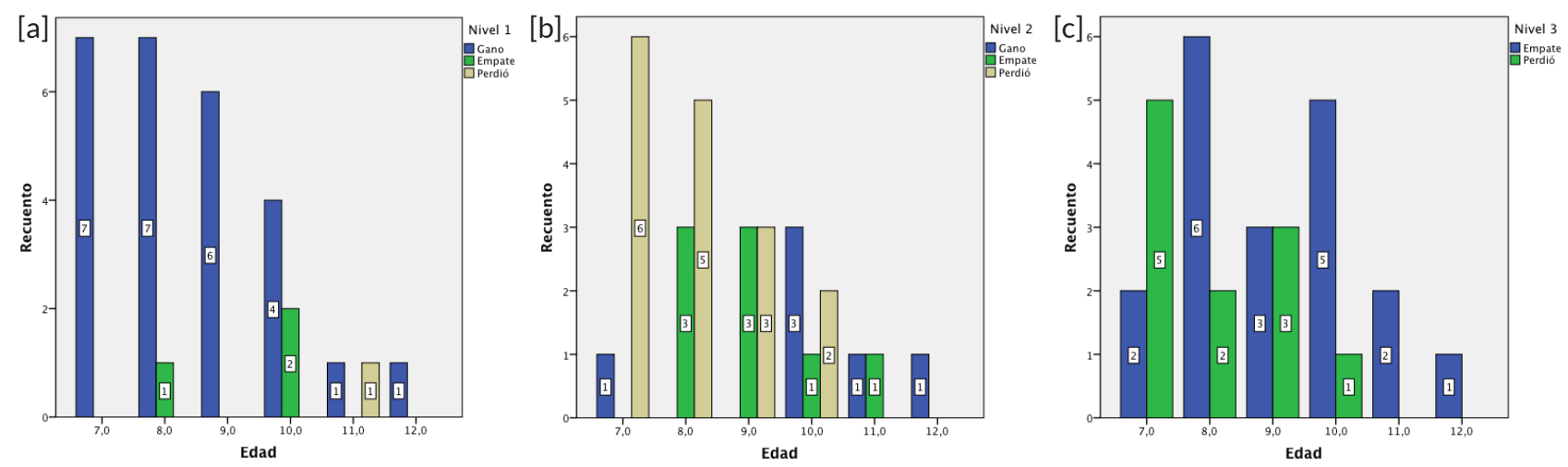

Gráfico 1. (a) Resultados de la Evaluación del juego Nivel 1. (b) Resultados de la Evaluación del juego Nivel 2. (c) Resultados de la Evaluación del juego Nivel 3.

Fuente: Elaboración propia.

Además se obtuvo una evaluación pedagógica en base a la matriz de evaluación de la Ficha Simplificada Catalogación y Evaluación de Programas Educativos del Dr. Pere Marqués de la Universidad Autónoma de Barcelona (2002). La Tabla 3 muestra los resultados obtenidos al justipreciar cada criterio de evaluación. Como se puede apreciar, existe una aceptación en todos los criterios evaluados por los docentes (Rango de aceptación de $0: 2$ ).

\begin{tabular}{lr}
\hline Criterio & F Test \\
\hline Aspectos pedagógicos y funcionales & 1,54 \\
Aspectos técnicos y estéticos & 1,75 \\
Recursos didácticos que utiliza & 1 \\
Esfuerzo cognitivo requerido & 1,43 \\
\hline
\end{tabular}

Tabla 2. Resultados del Test de Análisis de Confiabilidad.

Fuente: Elaboración propia.

\section{Discusión}

El reto de esta investigación, está dado para que los/as niñas se interesen por las actividades lúdicas de aprendizaje de orden significativos, desarrollando la meta-cognición en juegos virtuales y educativos. Con estos juegos se busca estimular el pensamiento lógico, desarrollando sus capacidades. Lo lúdico es altamente motivador, creativo, atractivo, divertido y muy cercano a la realidad. Si a esto le adicionamos la parte del aprendizaje matemático-lógico, se torna más eficaz. Esto conduce a pensar en los juegos educativos no solo como un entretenimiento o una diversión. Hay que pensar, más bien que en la actualidad se debe concienciar del potencial educacional de los juegos, los mismos que deben tener una estrategia metodológica y dinámica que influya positivamente en los/as niños/as. 


\section{TRABAJOS RELACIONADOS}

Durante la investigación se han encontrado una importante cantidad de trabajos relacionados con los videojuegos tipo Tres en Raya. A continuación se resume los revisados en este estudio:

Russell y Norvig (2002) proponen una solución basada en estrategias óptimas utilizando el algoritmo del MiniMax, donde Min tiene algo que decir y Max por tanto debe encontrar una estrategia contingente, que especifica el movimiento de Max en el estado inicial. Después los movimientos de Max en los estados que resultan de cada respuesta posible de Min de los anteriores movimientos, etc. Otros trabajos con diversas soluciones del juego de Tres en Raya son el algoritmo de búsqueda Alpha-Beta (Watson, 2008), algoritmos genéticos (Bhatt, 2008), redes neuronales (Grim et al., 2005), Inteligencia Artificial basada en estrategias (Chakraborty, 2009), entre los más conocidos. Trabajos más contemporáneos como el de Xu, Min, Zhu, \& Chen (2014), que proponen implementar un algoritmo genético para resolver el problema de reducción de atributos enfocado en múltiples objetivos, que involucren pruebas de costos de múltiples tipos. Para evaluar el rendimiento del algoritmo, se definen tres métricas, desde el punto de vista estadístico. Este trabajo se diferencia del nuestro, ya que en este se realizan análisis estadísticos sobre varios juegos usando un algoritmo genético, mientras que en el nuestro se utilizan diferentes tipos de heurísticas. En otro contexto, Bondre, Kapgate, \& Ponkshe (2014), proponen la implementación del juego del Tres en Raya, utilizando para el efecto conceptos avanzados de Procesamiento de Imágenes y Técnicas de Programación de Juegos. Se propone que el desarrollo del juego se base en la simplicidad, de modo que sea fácil de entenderlo y utilizarlo. Con este propósito se determina utilizar un Láser de luz roja y un sistema de visión por computadores y procesamiento de imágenes combinado con una cámara Web, para controlar la selección y posicionamiento de movimiento escogido por el usuario. Comparado con el nuestro, el desarrollado en este proyecto utiliza un rayo láser y una cámara Web para llevar el control del juego entre el usuario y la máquina. Gronli, Hansen, Ghinea, \& Younas (2014), proponen una implementación de una versión móvil del juego de Tres en Raya para realizar una comparación de aspectos claves de estudio de tres plataformas líderes en el mercado que son Androide (basada en Linux de Google), Windows Phone (de Microsoft), e iOS (de Apple). Nuestro trabajo se enfoca en la implementación del juego con fines didácticos.

\section{CONCLUSIONES Y TRABAJO FUTURO}

En esta investigación se optimizó el video juego de Tres en Raya. Para lograrlo se ha empleado OOHDM, para el diseño conceptual y navegacional de la aplicación, con lo cual se creó una interfaz de usuario interactiva y amigable. Para la implementación de algoritmos se aplicó técnicas heurísticas de Inteligencia Artificial para que el jugador simulado y controlado por la computadora, pueda emular la toma de decisiones por sí mismo, y esté en capacidad de enfrentar al usuario. El método numérico es inédito, y se basó en series numéricas finitas, con lo cual se redujo la dificultad de los algoritmos. Para la ejecución del juego se incorporó un agente virtual que brindó soporte al usuario en los diferentes niveles de dificultad. Las pruebas de evaluación del video juego, fueron realizados en una muestra estratificada y representativa de niños y niñas de 7 a 11 años. Los resultados muestran que este tipo de programas estimulan el desarrollo cognitivo de los niños que se encuentran en su etapa primaria de formación.

Como trabajo futuro se plantea el diseño y desarrollo del juego de Tres para ambientes 3D distribuidos enfocados a la educación y al entretenimiento, multi plataforma, que permiten integrar servicios de reconocimiento de voz o faciales (Speech or Facial Recognition). 


\section{REFERENCIAS}

Bello, R. (2002) Aplicaciones de la Inteligencia Artificial. Guadalajara: Universidad de Guadalajara, México.

Bhatt, A., Varshney, P., \& Deb, K. (2008). In search of no-loss strategies for the game of tic-tac-toe using a customized genetic algorithm. Proceedings of Genetic and Evolutionary Computation conference, Atlanta, USA, 889-896.

Bondre, C., Kapgate, D., \& Ponkshe, R. (2014). Laser Guided Tic Tac Toe. image, 6, 7.

Buckland, M. (2005). Programming game Al by example. Jones \& Bartlett Learning. Worware Game Developers Library. (1st ed.) USA.

Chakraborty, P. (2009) Artificial Intelligence Based Strategies to Play the Tic-Tac-Toe Game. Journal of Technology and Engineering Sciences. I 1, (1).

De Burgos J. (2013). Series Numéricas y de Potencias. García Maroto Editores.

Feuerstein, R., Klein, P.S., \& Tannenbaum, A. J. (Eds.). (1991). Mediated Learning Experience (MLE): Theoretical, psychosocial and learning implications. Freund Publishing House Ltd. England.

Grim, J., Somol, P., \& Pudil, P. (2005). Probabilistic neural network playing and learning Tic-Tac-Toe. Pattern recognition letters, 26(12), 1866-1873.

Gronli, T. M., Hansen, J., Ghinea, G., \& Younas, M. (2014, May). Mobile Application Platform Heterogeneity: Android vs Windows Phone vs iOS vs Firefox OS. IEEE International Conference on Advanced Information Networking and Applications, 2014, 28th, 635-641.
Lipman, M. (1998). Pensamiento complejo y educación (Vol. 43). Ediciones de la Torre.

Rich, E., \& Knight, K. (1994). Inteligencia artificial. (2nd ed.) McGraw Hill: México, 1994.

Russell S., \& Norvig, P., (2002). Artificial Intelligence:A modern approach. Pearson Education International. (2nd ed.), New Jersey - USA.

Schwabe, D., Rossi, G., \& Barbosa, S. D. (1996). Systematic hypermedia application design with OOHDM. Proceedings of the seventh ACM conference on Hypertext, 116-128.

Torres Vinueza, M. D., Fuertes, W., Villacís Silva, C. X., Zambrano Rivera, M. E., \& Prócel Silva, C. T. (2013). Puzzlemote: videojuego controlado con el mando de la Wii para niños de 6 a 10 años. AtoZ: novas práticas em informação e conhecimento, 2(2), 94-105.

Villacís, C., Fuertes, W., Bustamante, A., Almachi, D., Procel, C., Fuertes, S., \& Toulkeridis, T. (2014, October). Multi-player Educational Video Game over Cloud to Stimulate Logical Reasoning of Children. IEEE/ACM International Symposium Distributed Simulation and Real Time Applications, 2014, 18th, 129-137.

Vygotsky, L. S. (2000). El desarrollo de los procesos psicológicos superiores. Crítica. Grijalbo, Barcelona.

Xu, B., Min, F., Zhu, W., \& Chen, H. (2014). A Genetic Algorithm to Multi-objective Cost-sensitive Attribute Reduction. Journal of Computational Information Systems, 10(7), 3011-3022.

Watson M. (2008). Practical artificial intelligence programming with java. The MIT Press. (3rd ed.). Massachusetts, USA.

\section{Como citar este artigo (ABNT):}

VILLACÍS, C. J.; FUERTES, W. M.; BUSTAMANTE, C. A.; ZAMBRANO, M. E.; TORRES, E. P.; AULES, H. M.; TACURI, A. G.; BASURTO, M. O. Optimización del juego tres en raya con niveles de dificultad utilizando heurísticas de inteligencia artificial. AtoZ: novas práticas em informação e conhecimento, Curitiba, v. 3, n. 2, p. 95-106, jul./dez. 2014. Disponível em: <http://www.atoz.ufpr.br>. Acesso em:

\section{How to cite this article (APA):}

Villacís, C. J., Fuertes, W. M., Bustamante, C. A., Zambrano, M. E., Torres, E. P., Aules, H. M., Tacuri, A. G., \& Basurto, M. O. (2014). Optimización del juego tres en raya con niveles de dificultad utilizando heurísticas de inteligencia artificial. AtoZ: novas práticas em informação e conhecimento, 3(2), 95-106. Retrieved from http:// www.atoz.ufpr.br 